\title{
A New Qualification and Certification for Specialist ICT Teachers
}

\author{
Sigrid Schubert \\ Didactics of Informatics and E-learning, University of Siegen, D-57068 Siegen, Germany, \\ schubert@informatik.uni-siegen.de
}

\begin{abstract}
Student teachers qualify as specialist ICT teachers. This paper discusses selected objectives of informatics, the development of appropriate e-learning modules and some social and ethical requirements of such a contribution to teacher education. In 2001 and 2002 initiatives of IFIP and EU and selfreflection led to more collaborative learning and certification scenarios.
\end{abstract}

\section{INTRODUCTION}

Teachers worldwide have been surprised at the dramatic changes in their profession. When ICT first emerged in schools they expected a new learning and teaching medium which could be integrated with traditional pedagogical concepts. But the recent IFIP conference on the school of the future held in Chile (Taylor \& Hogenbirk, 2001) showed that the informatics core of ICT gave rise to new ways of learning and teaching. Therefore appropriate pedagogical concepts were to be created and evaluated. The contribution of the research group 'Didactics of Informatics', chaired by the author, is a verifiable suggestion for new ways of qualifying and certifying specialist ICT teachers. Members of the group were informatics scientists, teacher trainers, educationalists, students of informatics and student teachers. They were involved in a process over a period of four years:

- developing guidelines and curricula in Germany (section 2),

- training student teachers and evaluating courses at the Universities of Dortmund and Siegen (sections 3 and 4),

- designing e-learning material in national and international communities (sections 4 and 5),

The original version of this chapter was revised: The copyright line was incorrect. This has been corrected. The Erratum to this chapter is available at DOI: 10.1007/978-0-387-35701-0_35 
exchanging experiences at the IFIP conferences in Copenhagen 2001 and Dortmund 2002 (section 6).

In this paper, obstacles and recommendations are discussed.

\section{EDUCATIONAL GUIDELINES FOR SPECIALIST ICT TEACHERS}

Publications of IFIP (Mulder \& van Weert, 1998; van Weert \& Tinsley, 1994) inspired the development of educational guidelines for ICT teachers issued by the German Informatics Society in 1999. All student teachers were to have the opportunity to choose such certified courses of 300 training units (60 minutes each). The following six fields of informatics were compulsory:

1. The students should know social and ethical criteria and be able to evaluate connections between the Information Age and the impact of ICT.

2. They should know the process of modelling in informatics and be able to create and to evaluate such modelling solutions.

3. They should know how to solve problems with the help of informatics to achieve reliability, extendibility, reusability and portability.

4. They should know the basics of algorithms, data structures and programming languages, and be able to develop and to evaluate software.

5. They should know the basic concepts of information security and of data protection laws, and be able to apply this knowledge.

6. They should know the fundamental principles of informatics systems.

These aims are characteristic of a lot of extended training programmes in informatics. But in this case the time available was only 120 training units. Appropriate educational experiences and learning materials were not available. It was clear that we could not use the traditional learning and teaching methods. The courses were expected to provide the opportunity to study the subject areas in a learner-centred, interdisciplinary and projectoriented way with the right mixture of lectures, exercises, practical training periods and examinations.

On the basis of this curriculum the qualification started in October 2000 at different universities. At the University of Dortmund all student teachers were offered two new courses, 'Computing Systems' and 'Modelling in Informatics'. The first course enables future teachers to understand the functionality, architecture, application and administration of highly complex ICT systems in education. The second course was designed and held by the author's group. It helps teachers to be fully responsible members of an 
interdisciplinary collaborative working group for the evaluation, implementation, modification and creation of ICT systems in education.

\section{MODELLING IN INFORMATICS}

The participants in this course were experienced teachers from schools and student teachers from universities. They started with a discussion and choice of a project theme, for example an ICT-based learning aid for English lessons. Parallel with the introduction to fields $2-4$ (section 2), they developed their project programme and carried out its various phases. Each participant had his or her own project, but experienced teachers and student teachers worked together in mixed groups. This cooperation provided the opportunity to exchange the views of schools and universities and to test some ideas in the classroom practice. The educators made two decisions about the learning matter, one concerning the design strategy and the other concerning the programme language:

1. The introduction to modelling and problem solving is based on objectoriented design (OOD), which is suggested by cognitive aspects and which is state of the art in informatics.

2. The programme language was to stay in the background as a mere aid, easy to understand and to apply. That is why the script language Python has been chosen for object-oriented programming (OOP) as it offers a rich library of ready modules for the construction of software and brings the logical structure of solutions into focus.

But both of these decisions have also caused difficulties in the learning process.

The first problem is to learn OOD. Students start with easy tasks related to an ICT project 'library' and such object-oriented solutions are easy to discover and to describe but difficult to implement. An author, for example, normally writes more than one book. And a book may have more than one author. Beginners have no trouble identifying the informatics objects 'authors' and 'books' and to connect both with a correct relation, called association. But they can not cope with the creation of classes to produce the objects and the Python representation of association between the objects.

The second problem is to learn formalisation. This course brings various student teachers together. This results in the encounter of scientific cultures that are very different from the ways of creative thinking that are specific to informatics. Arts students, for example, get a shock when confronted with a formal language of informatics. Students of linguistics, on the other hand, are well able to read and write in such a formal language. In interdisciplinary 
teams both groups stand to gain from the wide range of previous knowledge and talents.

In the early stages an additional problem cropped up. On account of the complicated content and difficult methods of informatics a strong lobby argued that teachers need no deeper understanding of informatics to manage ICT at schools. Part of this lobby in Germany were influential enterprises which trained teachers to use their products. But the web-based learning and teaching approach at schools failed because specialist ICT teachers were not adequately educated (Watson \& Andersen, 2002). So it is necessary to explain the relation between modelling in informatics and web-based learning in more detail. Discussion with experienced teachers shows that the following tasks will have to be tackled by specialist ICT teachers in cooperation with the school administration:

- informatics consultation and training of colleagues,

- careful choice of applications for web-based learning and teaching,

- infrastructure decisions from pedagogical and informatics points of view,

- responsibility for information security and data protection at their schools.

Therefore modelling in informatics must be understood as the complete process of specification, design, programming and evaluation of informatics applications. This has to happen with an ICT developing environment for editing, saving, loading and executing individual software solutions.

Easy learning objectives are extended to more complicated ones. Students will, for example, discover the power of the concept of data structures step by step as the first ICT project, 'library,' requires numerous names of authors and titles of books modelled as strings. And someone must input all these data. Then the user can make inquiries to which the system can display the response. But there can be an emergency when the system loses the huge collection of data called data structure of records. The records can not be permanently saved in the main memory. So a storage medium is necessary to save the records in a more complex data structure called a file. In this way students understand the key concept of each database application and learn to control the access to files. Every file is connected with basic operations such as opening, reading, closing, creating, writing, deleting. A lot of files are not well protected. Many schools have no reliable concept of information security and privacy and no efficient backup mechanism as they have no specialist ICT teachers (Passey \& Kendall, 2002).

Software designers and users lose track of things without the highly successful principle of reducing complexity, the principle of modularity. Modularisation improves the flexibility and comprehensibility of an informatics system. The design of classes to create informatics objects, for 
example books and readers, is an opportunity to develop one's own modules. But modularisation also requires the ability to import existing modules of partial solutions from an informatics modules library. This sounds very easy, but the students have to learn how to prove the correct functionality of each module interface before they can use it. The module ensures information hiding of all internal data and access over the interfaces with parameters. The control and connection of parameters is difficult to understand, so that students need special support, for example with learning aids for exploration (Brinda \& Schubert, 2002b).

Records or, in general, all sequences of informatics objects can build up the more complex data structure list. The list is an important cognitive tool for problem solving, and the library software also needs some lists, like, for example, a list of books and a list of readers. Extendibility and reusability are two criteria for evaluating the quality of informatics systems that can be realised with the concept of inheritance. All special lists, for example, inherit attributes and operations, also called methods, from an abstract class list (Figure 1). The concept of inheritance is easy to understand but difficult to apply. Here the students need further learning aids.

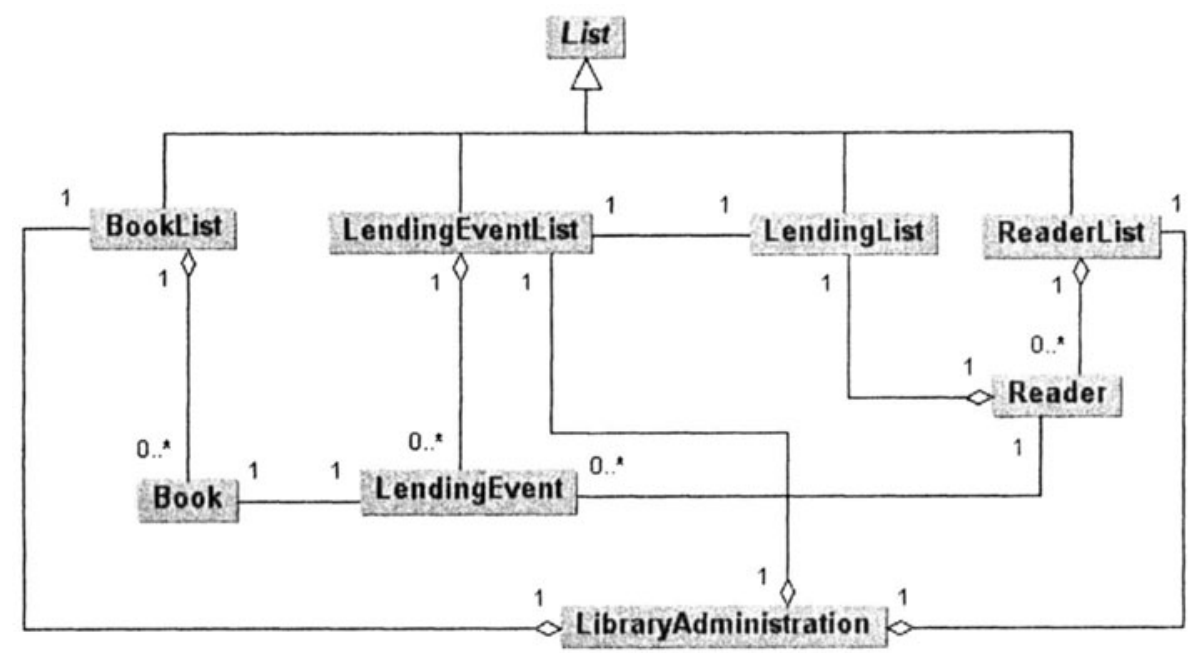

Figure 1. Class diagram of the library 


\section{EXPLORATION MODULES FOR OBJECT- ORIENTED MODELLING}

The students can successfully learn the development process of ICT solutions through approaches based on theories of constructivism. This requires learning material that allows a lot of student errors without a system collapse. The author has introduced the expression 'exploration modules (EMs)' for such a special learning software. EMs are basic components of the 'Didactic System for OOM', which has been implemented at the University of Dortmund (Brinda \& Schubert, 2002a). EMs allow the students to learn by discovery. EMs are difficult to programme and not available for all the basic skills in informatics which are connected with the process of OOM, like:

a. a deeper understanding of object-oriented problem solving,

b. OOD with the Unified Modelling Language (UML),

c. programming of OOD with the script language Python,

d. evaluation of educational results and self-assessment.

EMs support the objectives a. and b. while $c$. and d. have to be learned in the traditional way. Learners can design a class diagram in UML with different lists. EMs can examine the existence of the main classes and the relations among these classes. EMs do not show the tutor's solution but the results of the student's solution. So students have to discover the difference between their aims and their results. In difficult cases EMs give recommendations, such as to move the joint attribute 'number of elements' to the abstract class list (Figure 2).

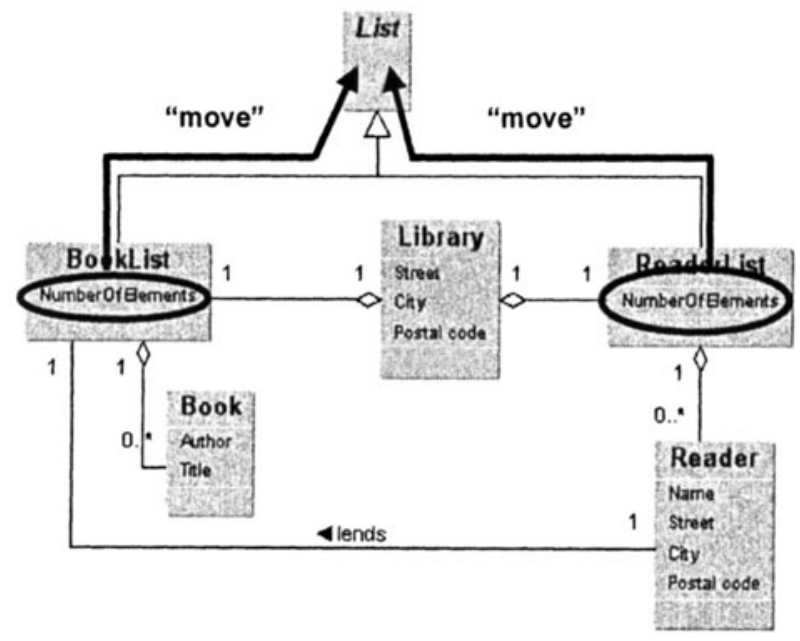

Figure 2. Exploration of inheritance 
A project team of informatics students has developed a collection of EMs and integrated them into a 'Learning Environment of OOM (LEO)'. With LEO the student teachers can start application scenarios, for example 'library', and watch different 'views'. There is a view of typical administration procedures represented by icons for service staff, readers with user cards, shelves with books, computers with catalogues and databases. The learners can switch over to more abstract views like object diagrams and class diagrams with UML to discover the process of informatics modelling through formalisation step by step. A big problem for beginners is to ensure completeness and consistency of an object-oriented model because in the past only programmes could be tested by computer. But EMs show the results of the model during the OOD stage. The learners are able to correct their errors immediately and do not have to stabilise the faulty structure until they reach the OOP stage. The complexity can be controlled when details are hidden or stressed. The learners can watch how messages are exchanged among objects, objects are created, used and destroyed. The change of object states and the execution of methods are monitored. EMs make the hidden processes of synchronisation and transformation of model parts visible, thus supporting the evaluation of models by the learners themselves. Empirical studies show the increasing interest of student teachers in presenting and discussing their models in groups.

\section{EUROPEAN CONSORTIUM OF INNOVATIVE UNIVERSITIES}

While other subjects have a long tradition in learning by discovery with suitable learning aids, informatics had first to develop high-quality learning aids. Such a complex task could be better solved with partners. So we have become part of national and international networks for cooperation, for example the European Consortium of Innovative Universities (ECIU, 2002). In cooperation colleagues from the Universities of Aalborg, Aveiro, Barcelona, Dortmund, Glasgow, Joensuu, Siegen and Twente began to develop webbased learning aids for informatics education. The author was project manager of the informatics track. She was responsible for the successful running of two workshops, the stimulation of the cooperative work for half a year, and the delivery of the final product. Starting in March 2002 with a first workshop discussing realistic aims, the team decided to work on two themes, recursion and programming paradigms. The group developed a joint statement for the design and structure of further e-learning material. A second workshop was held in July 2002 at the IFIP Conference on 'Social, Ethical and Cognitive Issues of Informatics and ICT (SECIII)' to share the elearning experience with a wider international community. 
We wanted to have e-learning support for programming paradigms because specialist ICT teachers need the ability to evaluate different paradigms but have time to learn only one paradigm in depth. The examples developed by the ECIU team were integrated in the course 'Modelling in Informatics'. The student teachers in Dortmund and Glasgow used them to discuss the pros and cons of different problem-solving strategies of informatics, for example the functional and the object-oriented programming paradigms. They also compared the given e-learning material with the results of their own ICT project. The students from Dortmund were encouraged to continue the course when they realised they were not behind the educational aims, as their international partners had to overcome the same obstacles and problems.

With members of the ECIU team, students discussed the recommended design approach to the creation of appropriate e-learning components as features within hybrid courses typical of schools. We reviewed the methodological process with the group of learners. The colleagues from Glasgow knew best how to produce animated components to be used with learning materials from the project called 'Scottish Teachers On-line Modules'. The animations afford learner interaction with scope to explore the behaviour of the represented example and allow the students to make some processes visible that cannot easily be discerned. This experience was stimulating for our students. Smaller learning components that could be used widely within existing course structures and across differing schools could be developed by students and ICT teachers.

\section{SOCIAL AND ETHICAL REQUIREMENTS}

In 1998 we entered upon group communication and annual meetings of students and ICT teachers to set up a social network of mentors to guide the classroom practice of beginners. During the following years examples of transferring first-rate practice experience were collected and evaluated. But how to implement social and ethical requirements within the educational process with ICT was an open question. Therefore we expected a paradigm shift from the results of the IFIP Conference SECIII which was held in Dortmund in July 2002 (van Weert \& Munro, 2003). We invited ICT teachers to participate in this conference and to set us thinking about the evolution of privacy over the course of centuries. The results of working group discussions (SECIII, 2002) were very helpful in redesigning courses at the University of Siegen. Now the students are responsible for the planning of their collaborative work with experienced teachers. Most of our students know mentors from their previous classroom practice terms. Students make a great effort in the social embedding of their ICT solutions. This has a long-term effect on the linking of cognitive and social aims. When a study group de- 
cides to develop web-based exercises for German lessons, including the assessment of students, they normally face a lot of social and ethical problems, such as the protection of privacy and the danger of masquerading. The mentors insist on security by means of encryption and authentication, together with control of access. This reality-based application has motivated the students to study the basic concepts of information security very carefully.

Today all institutions require a high level of infrastructure with complex computer networks and distributed systems. But the installation and management of such complicated informatics systems are not pedagogical tasks. Often school administration can not cope with these problems. So experienced teachers and student teachers have to face up to increased stress through the application of ICT in schools. The SECIII results helped us to understand that this problem can not be solved with new ICT devices but only with a new level of social and ethical relationships. Future specialist ICT teachers must be educated as producers rather than as consumers. The students have a certain impact on their partner schools because they do not insist on traditional procedures and have much more time for innovations. In all institutions the proper management of knowledge is a source of progress which can be made accessible only with difficulty. Human aspects of collaboration have to be taken into account, like the question of motivating successful teachers to support their colleagues. Their responsibility for the next generation of teachers should result in such a motivation. Therefore the joint training of students can help to promote knowledge management at schools and universities and between them, so that student teachers will become equal members of such a learning community.

The borderline between fact and fiction is fuzzy in ICT-supported learning environments and a lot of dangerous situations are hidden under colourful surfaces. Learners need orientation for their behaviour, decisions and responsibility that only social and ethical rules can provide. But such rules are learned when they are lived. Student teachers should have learned to avoid exaggeration. Not all e-mails, for example, need encryption, but in some cases encryption is the only reasonable way. If student teachers do not put this into practice in their daily lives they should not teach it. The change in the professional situation of teachers is extremely difficult because scientific content and pedagogical strategy are only effective in combination with the social and ethical behaviour of teachers appropriate to the Information Age. We have changed the subject content to promote the critical understanding of software agents as an attack on informational self-determination, an elementary human right. The students have acquired the ability to fight off cookies and user profiles successfully and to protect private data like photos and certificates. They have learned how to shield ICT environments with firewalls from software agents and to develop devices to demonstrate dan- 
gerous attacks to learners. They are convinced that learners need free access to web-based material to assume ethical responsibility.

We also changed the procedure of certification. New abilities are important and there is more flexibility of time management. It is no longer the product of an ICT project that is the focus of attention but the design process and the effectiveness of methods. This is why exams take place as group discussions with presentation, self-reflection and assessment of social integration of ICT and the impact on learning and teaching. The students present examples of ICT-supported learning environments that are theoretically well-founded and have been tested in classroom practice. This requires sufficient time for empirical studies with learners, which can be obtained either by putting the provisional results to test at school and redesigning them continuously, or by appending the empirical studies to the university courses.

\section{CONCLUSION}

The students were satisfied with their qualification as ICT teachers. They received excellent job offers and are involved in the continuous training of their colleagues. They use ICT to maintain their collaborative efforts in social networks after finishing their university training. Quite a number of them have become active members of professional communities of the German Informatics Society, to improve learning conditions with ICT in schools.

The course 'Modelling in Informatics' has met the objectives. The students were able to learn and teach in different age groups with teachers, parents and children and with students and teachers of school partnership programmes with different countries. The demand for such courses will increase. A lot of interdisciplinary research is necessary for better professional strategies of teachers in general and ICT teachers in particular. Today student teachers have to become involved in this change of learning and teaching paradigms step by step without being given a ready-made blueprint.

We plan to offer additional workshops for e-learning. They will provide opportunities for presenting and discussing new results and for developing methodologies among researchers, students and partners. Partners can present results of e-learning processes and new requirements. Students can choose themes, tasks and partners for their project work or diploma. 


\section{REFERENCES}

Brinda, T. \& Schubert, S. E. (2002a). Didactic system for object-oriented modelling. In D. Watson \& J. Andersen (Eds), Netvorking the Learner. Computers in Education (pp. 473-482). Boston: Kluwer Academic Publishers.

Brinda, T. \& Schubert, S. (2002b). Learning aids and learners' activities in the field of objectoriented modelling. In D. Passey \& M. Kendall (Eds), TelE-Learning: The Challenge for the Third Millennium. Boston: Kluwer Academic Publishers.

ECIU (European Consortium of Innovative Universities) (2002). Web-supported learning pilots. Retrieved October 6, 2002 from http://cvu.strath.ac.uk/eciu/

Mulder, F. \& van Weert, T. (Eds) (1998). Informatics in Higher Education. London: Chapman \& Hall.

Passey, D. \& Kendall, M. (Eds) (2002). TelE-LEARNING. The Challenge for the Third Millennium. Boston: Kluwer Academic Publishers.

SECIII (Social, Ethical and Cognitive Issues of Informatics and ICT). Working group discussions. Retrieved October 6, 2002 from http://wwwedu.ge.ch/cptic/prospective/ projets/ifip/workarea/dortmund/welcome.html).

Taylor, H. \& Hogenbirk, P. (Eds) (2001). Information and Communication Technologies in Education. The School of the Future. Boston: Kluwer Academic Publishers.

van Weert, T. \& Tinsley, D. (Eds) (1994). Informatics for Secondary Education - A Curriculum for Schools. Paris: Unesco. Retrieved October 6, 2002 from http://wwwedu.ge.ch/cptic/prospective/projets/unesco/1994/

van Weert, T. \& Munro, B. (Eds) (2003). Social, Ethical and Cognitive Issues of Informatics and ICT. Boston: Kluwer Academic Publishers.

Watson, D. \& Andersen, J. (Eds) (2002). Netvorking the Learner. Computers in Education. Boston: Kluwer Academic Publishers. 